\title{
Autoeficácia acadêmica e autorregulação da aprendizagem: rede de relacionamento em bases online
}

\author{
Maria Cristina Rodrigues Azevedo Joly ${ }^{1}$ \\ Universidade de Brasília, Brasília-DF, Brasil \\ Alexandre Luiz de Oliveira Serpa \\ Hogrefe Cetepp, São Paulo-SP, Brasil \\ Lisandra Borges \\ Universidade São Francisco, Itatiba-SP, Brasil \\ Rosana Maria Mohallem Martins \\ Centro Universitário de Itajubá - FEPI, Itajubá-MG, Brasil
}

\section{RESUMO}

O estudo refere-se à análise da produção científica em língua portuguesa sobre os construtos Autoeficácia acadêmica e Autorregulação da aprendizagem no período de janeiro de 2000 a dezembro de 2014 em bases de dados Redalyc e CAPES, na subárea Psicologia que contou com 43 itens. Foi realizada análise cientométrica a partir das informações dos artigos, e o escalonamento multidimensional e a rede de relacionamentos entre os autores foi feita a partir das referências. Os resultados evidenciaram que a maior parte dos artigos se refere à autorregulação e nestes a Teoria Social Cognitiva é a perspectiva teórica predominante. Quanto às referências, encontrou-se um total de 901 citações de 735 autores diferentes, sendo a maior parte destas procedentes de artigos científicos. A análise das redes apresentou o padrão de relacionamento entre eles e o alinhamento com cada um dos temas pesquisados.

Palavras-chave: cientometria; produção científica; indicadores científicos.

\section{ABSTRACT - Academic self-efficacy and learning self-regulation: Relationship network at online databases}

The aim of this study was to produce an analytical review of the scientific production in Portuguese about the constructs of academic self-efficacy and learning self-regulation between January 2000 and December 2014. The data consists of 43 manuscripts within the Psychology division of the Redalyc and CAPES databases Scientometric analysis was conducted based on information within the manuscripts and the multidimensional scaling and the network of relationships among the authors was conducted from the references. The results showed that the majority of articles refer to self-regulation and among these, Cognitive Social Theory is the dominant theoretical perspective. As for the references, a total of 901 citations from 735 different authors were found, with the majority coming from scientific articles. The networks analysis presented a pattern of relationship between them and alignment with each of the surveyed subjects.

Keywords: scientometrics; scientific production; scientific indicators.

RESUMEN - Autoeficacia académica y la autorregulación del aprendizaje: las redes sociales en las bases de datos El estudio se refiere al análisis de la literatura científica en idioma porugués, sobre los constructos autoeficacia académica y autoregulación del aprendizaje a partir de enero de 2000 a diciembre de 2014 en bases de datos Redalyc y CAPES, en la subárea Psicología, y contó con 43 ítems. Fue realizado análisis cienciométrica a partir de las informaciones de los artículos y el escalonamiento multidimensional, y la red de relacionamientos entre los autores se hizo a partir de las referencias. Los resultados mostraron que la mayoría de los artículos se refieren a la autoregulación y en estos la Teoría Social Cognitiva es la perspectiva teórica dominante. En cuanto a las referencias, se encuentran un total de 901 citaciones de 735 autores diferentes, siendo la mayoría de ellos procedentes de artículos científicos. El análisis de las redes presentó el modelo de relacionamento entre ellos y el alineamiento con cada uno de los temas investigados.

Palabras-clave: cienciométrica; producción científica; indicadores científicos.

A autoeficácia é considerada como uma crença que o indivíduo tem sobre sua capacidade de desempenho em atividades específicas, envolvendo, para isso, o julgamento sobre suas competências (Zimmerman, 2000). Considerando-se o domínio específico de interesse, segundo Bandura (1993), a Autoeficácia acadêmica 
refere-se à crença do estudante em sua capacidade de organizar e executar ações referentes às atividades e exigências acadêmicas, estando relacionada às habilidades cognitivas imbricadas na construção de novos conhecimentos, levando em conta, inclusive, as mudanças tecnológicas da atualidade (Joly \& Prates, 2011).

A relevância desse construto está na possibilidade de predizer a ação dos estudantes em função das crenças que têm em suas capacidades, mais do que pelo que, realmente, são capazes de realizar. Para Bandura (1993), os estudantes com alta crença de eficácia acadêmica estão mais disponíveis para os estudos e são mais persistentes diante dos desafios acadêmicos. Desse modo, mesmo que possuam modestas habilidades para o desempenho, se acreditarem em sua capacidade, poderão alcançar bons resultados. Pajares e Olaz (2008) comprovaram essa premissa quando, em seu estudo, identificaram que estudantes que acreditam em sua capacidade de bom desempenho previam resultados acadêmicos bem-sucedidos, independentemente de sua habilidade cognitiva.

Outro construto relacionado que fornece pistas sobre os métodos e os caminhos para a aprendizagem efetiva é a Autorregulação. Ele consiste em pensamentos, sentimentos e ações autogerados que são planejados e sistematicamente adaptados, conforme a necessidade para afetar a própria aprendizagem e motivação (Zimmerman, 2000). Na literatura, destacam-se várias abordagens sobre autorregulação na aprendizagem, como a comportamentalista, volitiva, construtivista, fenomenológica, vygotskiana e sociocognitiva de acordo com análise feita por Zimmerman (1989). Na perspectiva da teoria social cognitiva, Bandura (1991) define o construto autorregulação como um processo consciente e voluntário, o qual possibilita ao indivíduo gerenciar os próprios comportamentos, pensamentos e sentimentos para obtenção de metas pessoais. Desse modo, é considerado como um construto com muitas facetas, pois envolve processos cognitivos, como o automonitoramento, julgamentos autoavaliativos e autorreações.

Schunk e Ertmer (2000) apontam a reciprocidade entre os construtos ao afirmar que alunos com bom nível de Autoeficácia são mais motivados para utilizar os processos de autorregulação. Assim, a crença de Autoeficácia é um fator que pode contribuir para a utilização de estratégias autorreguladas de aprendizagem, como estabelecimento de metas e objetivos, seleção e adoção de estratégias de aprendizagem. Sendo assim, os alunos autorregulados têm maiores chances de se tornarem mais confiantes no seu sucesso acadêmico, aumentando a crença de Autoeficácia. Desse modo, pode-se considerar a autorregulação como uma das fontes de autoeficácia, pois permitirá ao estudante maiores experiências de sucesso (Bandura, 1997; Casanova, Azzi, \& Dantas, 2010; Schunk, 1995; Zimmerman, 2013).

Compreender o modo como o conhecimento se organiza e conhecer os autores que se destacam como eixos integradores dos temas circulantes é fundamental para o enfrentamento das dificuldades no processo de ensino e aprendizagem (Lopez Lopez, Silva, GarciaCepedro, Aguilar-Bustamante, \& Lopez, 2010), tornando possível identificar com clareza a origem epistemológica e ontológica que fundamentam um dado conceito ou área de conhecimento. Assim, identificar estudos disponíveis acerca do papel da aprendizagem na cognição e motivação, da influência das diferenças individuais e culturais no processo de autoeficácia e autorregulação, bem como estudos sobre avaliação e intervenção é de grande importância (Caprara et al., 2008; Joly et al., 2014; Polydoro \& Azzi, 2009; Rosário, 2001; Usher \& Pajares, 2007).

A avaliação psicológica educacional é destacada pela sua contribuição para que programas de intervenção sejam desenvolvidos e, assim, as pessoas possam ter acesso a uma educação de qualidade (Knijnik, Giacomoni, Zanon, \& Stein, 2014). Pesquisas que objetivam estudar construtos importantes da psicologia educacional como os abordados neste estudo e, principalmente, como esses construtos têm sido abordados na comunidade científica, contribuem para o desenvolvimento e aprimoramento de medidas que possibilitem a verificação do desempenho dos alunos.

No Brasil, o uso de técnicas bibliométricas quantitativas com base no mapeamento ou na investigação das redes de relacionamento ainda é pouco frequente, sendo que a maior parte da produção privilegia o uso das estatísticas descritivas (Neiva \& Torres, 2011). Este artigo apresenta um estudo de revisão de literatura que utilizou o conceito de estrutura cognitiva apresentado na teoria de análise de rede social, para analisar o levantamento realizado, em repositórios brasileiros online de periódicos científicos, acerca da produção científica em língua portuguesa sobre autoeficácia acadêmica e autorregulação da aprendizagem.

A cientometria ou cienciometria é um campo de estudos que emergiu nas décadas de 1970 e 1980 como uma área que buscava se valer de métodos quantitativos, com o objetivo de compreender o desenvolvimento da produção científica e, em conjunto, debater os caminhos das políticas em ciência das nações (Chen, Mccain, White, \& Lin, 2002). O enfoque está no levantamento de índices de impacto, produção e desenvolvimento das publicações, além da investigação dos padrões das especialidades e áreas subscritas à produção científica. As análises cientométricas geralmente se utilizam da informação sobre número de citações para ordenar as revistas, trabalhos ou autores mais relevantes de determinado campo (Leydesdorff, 2011) e definir os avanços científicos pertinentes à área de conhecimento (Poblácion, Witter, \& Silva, 2006; Witter, 1999).

Também são comumente empregados métodos pictográficos para a representação espacial dos relacionamentos entre as categorias analisadas, cujos estudos são 
referidos como pertencentes à subárea denominada cartografia da ciência. Esses mapas ou redes são mais utilizados para a representação da distribuição de conteúdo de uma área de conhecimento específica ou de um conjunto de autores, revistas e outros atributos, além da intensidade da relação que conservariam entre si. A posição dos conteúdos é determinada pela distância em que eles estão representados no espaço (Noyons, 2001). Sua relevância está em desvelar as interconexões entre as produções e as influências teórico-epistemológicas de modo a facilitar a compreensão do desenvolvimento de um referido conceito ou campo de conhecimento.

Levando-se em conta a os pressupostos da cienciometria e o mapeamento das redes de relacionamento científico definidas pelas citações como proposto por Leydesdorff (2008), o objetivo do presente estudo foi mapear o conjunto de citações da produção ligada à autoeficácia acadêmica e à autorregulação da aprendizagem de modo a possibilitar a identificação dos autores que mais são referenciados na produção científica da área, especificamente na psicologia brasileira e de países de língua portuguesa. Ao lado disso, buscou-se caracterizar a rede de relações entre os autores que publicam em língua portuguesa sobre a temática, considerando o ano da publicação; os construtos avaliados, autoeficácia, autorregulação ou ambos; escolaridade da amostra; objetivos dos estudos, se referia à avaliação ou à intervenção; delineamento do estudo; perspectiva teórica e a revista em que foi publicado o artigo.

\section{Método}

\section{Amostra}

O estudo iniciou com a busca dos artigos, a qual foi efetuada na base de dados Redalyc e em todas as que compõem o portal de periódicos da Coordenação de Aperfeiçoamento de Pessoal de Nível Superior (CAPES), na área de conhecimento Ciências Humanas e subárea Psicologia. As palavras-chave utilizadas foram "autorregulação da aprendizagem" e "autoeficácia acadêmica", levando em consideração a escrita anterior e posterior ao novo acordo ortográfico da língua portuguesa (auto-regulação; auto-eficácia). Foram selecionados os artigos que continham, ao menos, o resumo e as referências por se considerar como critério mínimo de inclusão para viabilizar a análise cientométrica (Poblácion et al., 2006). Todos os resultados foram analisados, posteriormente, contemplando os demais critérios de inclusão: texto em língua portuguesa; data de publicação referente ao período de janeiro 2000 a dezembro de 2014 e trabalhos realizados na área educacional.

As informações foram organizadas em uma base de dados que continha elementos que caracterizavam os artigos e todas as referências utilizadas pelos autores de cada publicação. Foram relevantes o ano da publicação; os temas autorregulação, autoeficácia ou ambos; perspectiva teórica; o delineamento do estudo, teórico ou empírico; o objetivo, se se referia à avaliação ou à intervenção; o nível ou etapa de ensino em que se situava a amostra e o país em que foi realizado o estudo, como indica o procedimento de análise pela metaciência (Witter, 1999) e cientometria (Poblácion et al., 2006).

\section{Procedimento e Análise de Dados}

As análises estatísticas descritivas caracterizaram a produção científica relacionada aos construtos pesquisados. Em seguida, o foco foi definir o mapeamento da rede de relacionamento entre as citações. Os três primeiros autores, em citações com mais de três autores, foram considerados para o mapeamento. Esse corte tem como propósito evitar um excesso de dados faltantes na matriz de dados que será analisada, bem como identificar e trabalhar com aqueles autores que tenham mais contribuído com o manuscrito. Definidos os critérios, foram identificados os autores mais frequentemente citados no total de referências a fim de gerar a matriz de correlações cosine para calcular a distância relativa entre os elementos de uma matriz por meio do cosseno entre elas, seguindo a abordagem recomendada por Leydesdorff (2007; 2008; 2011) e Salvador e López-Martínez (2000). Para o estudo da estrutura subjacente à matriz, inicialmente, calculou-se o número de fatores possivelmente existentes com a análise paralela ${ }^{2}$, valendo-se tanto do método de componentes principais quanto do de fatoração do eixo principal. Frente aos resultados obtidos, as dimensões encontradas foram interpretadas, tomando como referência o fato de que cada dimensão representaria uma área de conhecimento ou disciplina.

Em seguida, procedeu-se o uso do escalonamento multidimensional. Essa técnica é utilizada para a produção de um mapa que descreve a relação que determinados objetos conservam, baseando-se na distância que eles mantêm entre si. No presente trabalho, foi usada a distância euclidiana (Manly, 2008), como medida indicativa de quais citações aparecem comumente em conjunto, o que permitiu mapear as áreas e os principais autores que a compõem, definindo, assim, as redes de relacionamento. Nestas, os coautores, ou seja, os que estão referenciados nos mesmos artigos estão próximos e diretamente ligados pelas setas. Os mais citados ocupam as posições centrais da rede e são orbitados pelos outros autores e, assim, é possível vislumbrar aqueles que se destacam em determinada disciplina ou área de conhecimentos, bem como os que representam o elo entre elas.

${ }^{2}$ Ver detalhes em: Hom, J. L. (1965). A rationale and test for the number of factors in factor analysis. Psychometrica, 30(2), 179-185. 
As Tabelas de frequência foram geradas no software SPSS ${ }^{\circledast}$ (Statistical Package for the Social Sciences) versão 19.0. As demais análises foram realizadas pelo software livre R (R Development Core Team, 2012), com os pacotes psych (Revelle, 2012), lsa (Wild, 2011) e network (Butts, Handcock, \& Hunter, 2012).

\section{Resultados e Discussão}

Aplicando os critérios de inclusão na amostra, foram analisados 43 artigos, sendo 23 com a palavra-chave "autorregulação da aprendizagem", 16 com "autoeficácia acadêmica" e quatro com ambos os termos. A Tabela 1 apresenta as características gerais dos artigos. O maior número de publicações contempla estudos empíricos, focados na avaliação dos construtos e, que em sua maioria, realizados com estudantes do ensino fundamental, considerando-se os brasileiros e portugueses. A presença de valores missing nas dimensões pesquisadas ocorreu, principalmente, pela indefinição de público-alvo, objetivo e perspectiva teórica.

Tabela 1

Frequências e Percentual das Publicações Científicas Relacionadas aos Construtos Autoeficácia Acadêmica e Autorregulação da Aprendizagem

\begin{tabular}{|c|c|c|}
\hline Conteúdo analisado & $f$ & $\%$ \\
\hline \multicolumn{3}{|l|}{ Tipo de estudo } \\
\hline Teórico & 11 & 25,6 \\
\hline Empírico & 32 & 74,4 \\
\hline Total & 43 & 100 \\
\hline \multicolumn{3}{|l|}{ Objetivo } \\
\hline Intervenção & 12 & 29,3 \\
\hline Avaliação & 29 & 70,7 \\
\hline Total & 41 & 100 \\
\hline \multicolumn{3}{|l|}{ Etapa de ensino } \\
\hline Fundamental & 13 & 33,3 \\
\hline Médio & 4 & 10,3 \\
\hline Básico ${ }^{a}$ & 13 & 33,3 \\
\hline Superior & 9 & 23,1 \\
\hline Total & 39 & 100 \\
\hline \multicolumn{3}{|l|}{ País } \\
\hline Brasil & 17 & 39,5 \\
\hline Portugal & 26 & 60,5 \\
\hline Total & 43 & 100 \\
\hline \multicolumn{3}{|l|}{ Construto } \\
\hline Autoeficácia & 16 & 37,2 \\
\hline Autorregulação & 23 & 53,5 \\
\hline Ambos & 4 & 9,3 \\
\hline Total & 43 & 100 \\
\hline \multicolumn{3}{|l|}{ Perspectiva teórica } \\
\hline Didática metacognitiva Monereo & 1 & 2,56 \\
\hline Modelo ACT & 1 & 2,56 \\
\hline Modelo do adaptable learning & 1 & 2,56 \\
\hline Modelo SAL (students approaches to learning) & 1 & 2,56 \\
\hline Teoria do processamento da informação & 1 & 2,56 \\
\hline Revisão de múltiplas teorias & 2 & 5,13 \\
\hline Teoria social cognitiva & 32 & 82,05 \\
\hline Total & 39 & 100,0 \\
\hline
\end{tabular}

Nota. ${ }^{a}$ A despeito das diferenças entre a denominação de ensino básico entre Brasil e Portugal, a terminologia aqui aplicada se refere à conceituação brasileira - ensino fundamental e médio. 
Quanto ao objetivo dos estudos, predominaram as publicações que enfatizam a avaliação $(70,7 \%)$, em detrimento da intervenção (29,3\%). Polydoro e Azzi (2009) sugerem o refinamento das medidas de avaliação, além de delineamentos longitudinais e causais ao constatarem dados semelhantes aos do presente estudo. Considerando que a autorregulação é um construto que pode promover mudanças no processo de aprendizagem, as autoras ressaltam a necessidade de investimento em estudos sobre intervenção para melhor caracterizar a influência das variáveis identificadas no processo avaliativo para a aprendizagem.

Constatou-se que a autorregulação é o tema principal de $53,5 \%$ dos artigos pesquisados e $37,2 \%$ tem como foco a autoeficácia e, em apenas 9,3\% dos estudos, há o relacionamento entre ambos os construtos. Esses resultados ratificam as análises feitas por Maciel (2010), bem como se deve considerar Schunk e Ertmer (2000), os quais apontavam os efeitos recíprocos entre os construtos. Nessa perspectiva, cabe destacar que, para atingir um bom nível de autoeficácia, é necessário a exposição do aluno a situações em que este obtenha um feedback positivo sobre o seu desempenho, ao mesmo tempo, para o desenvolvimento da autorregulação, o aluno deve se sentir autoeficaz. Assim, faz-se necessário ampliar o número de estudos em que haja a relações entre os dois construtos para melhor caracterizar as variáveis presentes e controladoras do contexto de aprendizagem, de acordo com a perspectiva de vários pesquisadores expertos no tema (Blickle, 1996; Boekaerts, 1996; Bzuneck, 2005; Castro, 2007; Choi, 2005; Eilam, Zeidner, \& Aharon, 2009; Flavell,1979; Guimarães \& Boruchovitch, 2004; Lopes da Silva \& Sá, 1993; Mayer, 2011; Paris \& Winograd, 1990; Valle et al., 2009).

Enfatizando a perspectiva teórica adotada pelos artigos, observou-se que a Teoria Social Cognitiva foi o modelo teórico de $82 \%$ das publicações analisadas, apesar das diversas perspectivas teóricas acerca da autorregulação, como discute Zimmerman (2001; 2013). Tal fato, de acordo com o autor, refere-se à contemporaneidade da síntese teórica desse modelo que valoriza a interação entre o sujeito, o meio e o comportamento. Há também que se considerar, de acordo com Zimmerman (2013), a relação entre os construtos, sendo a autorregulação motivada pela autoeficácia e pelos resultados da realização.

Tabela 2

Distribuição das Frequências e Percentual das Citações por Tipo de Publicação

\begin{tabular}{lccc}
\hline & Tipo de publicação & $f$ & $\%$ \\
\hline Artigo científico & & 743 & 50,8 \\
Livro & 279 & 19,1 \\
Capítulo de livro & 300 & 20,5 \\
Tese ou dissertação & 59 & 4,0 \\
Outros & 82 & 5,6 \\
Total & 1463 & 100,0 \\
\hline
\end{tabular}

Com relação às referências dos 43 artigos, encontrou-se um total de 1463 citações, como pode ser observado na Tabela 2. A metade delas é formada por artigos científicos $(50,8 \%)$, enquanto os capítulos de livros $(20,5 \%)$ e livros $(19,1 \%)$ vêm em seguida. Tal resultado é esperado na ciência, já que os artigos são o meio de comunicação das inovações e atualidades da área e de fácil acesso, especialmente nas bibliotecas virtuais, enquanto os livros apresentam um recorte do conhecimento consolidado em determinada época (Chen et al., 2002).

As publicações mais referenciadas em cada área, em função do tipo de publicação, estão apresentadas na Tabela 3. Dentre as revistas, o Journal of Educational Psychology foi citado em 56 oportunidades, seguida pelo Educational Psychologist, com 45 alusões. Uma análise cautelosa revela que quase a totalidade das revistas tem como tema a Psicologia Educacional, fato esperado, considerando que os construtos pesquisados são mais diretamente relacionados a essa área da Psicologia. Ademais, considerando os artigos, os livros e os capítulos de livro, as referências são basicamente estrangeiras, havendo pouca frequência de citações da produção em língua portuguesa da área. Polydoro e Azzi (2009), em sua análise acerca da produção brasileira, consideraram a importância de estudos que atentem para as especificidades e necessidades da realidade brasileira dada as características e diversidades da educação no Brasil em relação a países estrangeiros.

A análise da autoria das 1463 publicações apontou para 712 autores diferentes, sendo que B. J. Zimmerman é o mais referenciado, com 118 citações; seguido de P. S. L. F. Rosário com 91; A. Bandura com 80 e D. H. Schunk com 48, como pode ser observado na Tabela 4. Entre os mais citados, os autores brasileiros que se destacam são A. Lopes da Silva, S. R. Loureiro, L. Faria, P. C. Medeiros, E. Boruchovitch; M. B. M. Linhares e S. Polydoro. 
Tabela 3

Frequência dos Trabalhos mais Citados em Função do Tipo de Publicação

\begin{tabular}{|c|c|}
\hline Artigos & $f$ \\
\hline A social cognitive view of self-regulated academic learning & 7 \\
\hline Perceived self-efficacy in cognitive development and functioning & 7 \\
\hline Becoming a self-regulated learner: an overview & 6 \\
\hline Cultivating competence, self-efficacy, and intrinsic interest through proximal self-motivation & 6 \\
\hline Social cognitive theory: an agentic perspective & 6 \\
\hline Investigating self-regulation and motivation: historical background, methodological developments & 5 \\
\hline Self-efficacy beliefs in academic settings & 5 \\
\hline Processos de autorregulação da aprendizagem e realização escolar no ensino básico & 5 \\
\hline Revistas & $\mathrm{f}$ \\
\hline Journal of Educational Psychology & 56 \\
\hline Educational Psychologist & 45 \\
\hline Contemporary Educational Psychology & 20 \\
\hline American Psychologist & 17 \\
\hline Review of Educational Psychologist & 15 \\
\hline British Journal of Educational Psychology & 14 \\
\hline Journal of Personality and Social Psychology & 14 \\
\hline Livros & $\mathrm{f}$ \\
\hline Social foundations of thought and action: A social cognitive perspective & 14 \\
\hline Estudar o estudar: as (des)venturas do testas & 12 \\
\hline Self-efficacy: The exercise of control & 12 \\
\hline Self-regulated learning and academic achievement: Theoretical perspectives & 5 \\
\hline Aprendizagem autorregulada pelo estudante: perspectivas psicológicas e educacionais & 4 \\
\hline A formação social da mente & 4 \\
\hline Developing self-regulated learners: Beyond achievement to self-efficacy & 4 \\
\hline Handbook of self-regulation & 4 \\
\hline Saber estudar e estudar para saber & 4 \\
\hline Capítulos de livros & $\mathrm{f}$ \\
\hline Attaining self-regulation:A social cognitive perspective & 14 \\
\hline Developing self-fulfilling cycles of academic regulation: An analysis of exemplary instructional models & 6 \\
\hline Social cognitive theory and self-regulated learning & 6 \\
\hline O conhecimento estratégico e a autorregulação da aprendizagem: implicações em contexto escolar & 5 \\
\hline The role of goal orientation in self-regulated learning & 5 \\
\hline Theories of self-regulated learning and academic achievement: An overview and analysis & 5 \\
\hline Volitional aspects of self-regulated learning & 5 \\
\hline
\end{tabular}

Para conhecer os relacionamentos e caracterização das áreas de conhecimento, focou-se nos 27 autores referidos na Tabela 4. Calculou-se, então, a matriz de correlações cosine entre os autores e procedeu-se a análise paralela, que indicou a presença de dois fatores quando utilizado o método de fatoração do eixo principal e dois componentes, por meio do método de componentes principais, ou seja, foram encontradas duas dimensões subjacentes aos autores por ambos os métodos. Essas duas dimensões explicam aproximadamente $62 \%$ da variância dos dados. O resultado vai ao encontro do esperado, já que dois conceitos foram pesquisados, mas, apesar disso, quase $40 \%$ da variância dos dados ainda parece vinculada a outros temas de pesquisa dos autores, indicando que eles não se dedicam exclusivamente à autoeficácia, à autorregulação ou a ambos. Esse resultado confirma também os achados de vários estudiosos (Caprara et al., 2008; Polydoro \& Azzi, 2009; Rosário, 2001; Usher \& Pajares, 2007) acerca da pouca produção científica relativa aos dois construtos e indicam as temáticas de investigação mais relevantes. Assinalam, ainda, a relevância de estudos pautados na cientometria a fim de serem identificadas as lacunas na ciência como discutem Chen et al. (2002).

Na Figura 1, são apresentados os autores e as distâncias relativas entre eles, com o auxílio do escalonamento multidimensional. O foco é a observação da forma como os autores se organizam em função de cada 
conceito, conservando mais ou menos afinidade com cada um. Cada coordenada se refere a uma dimensão dos dados, ou seja, a um dos conceitos. Assim, a coordenada 1 é a dimensão do conceito de autoeficácia, e a coordenada 2 é a dimensão do conceito autorregulação da aprendizagem.

Tabela 4

Frequência de Autores mais Referenciados nas Publicações Científicas Analisadas

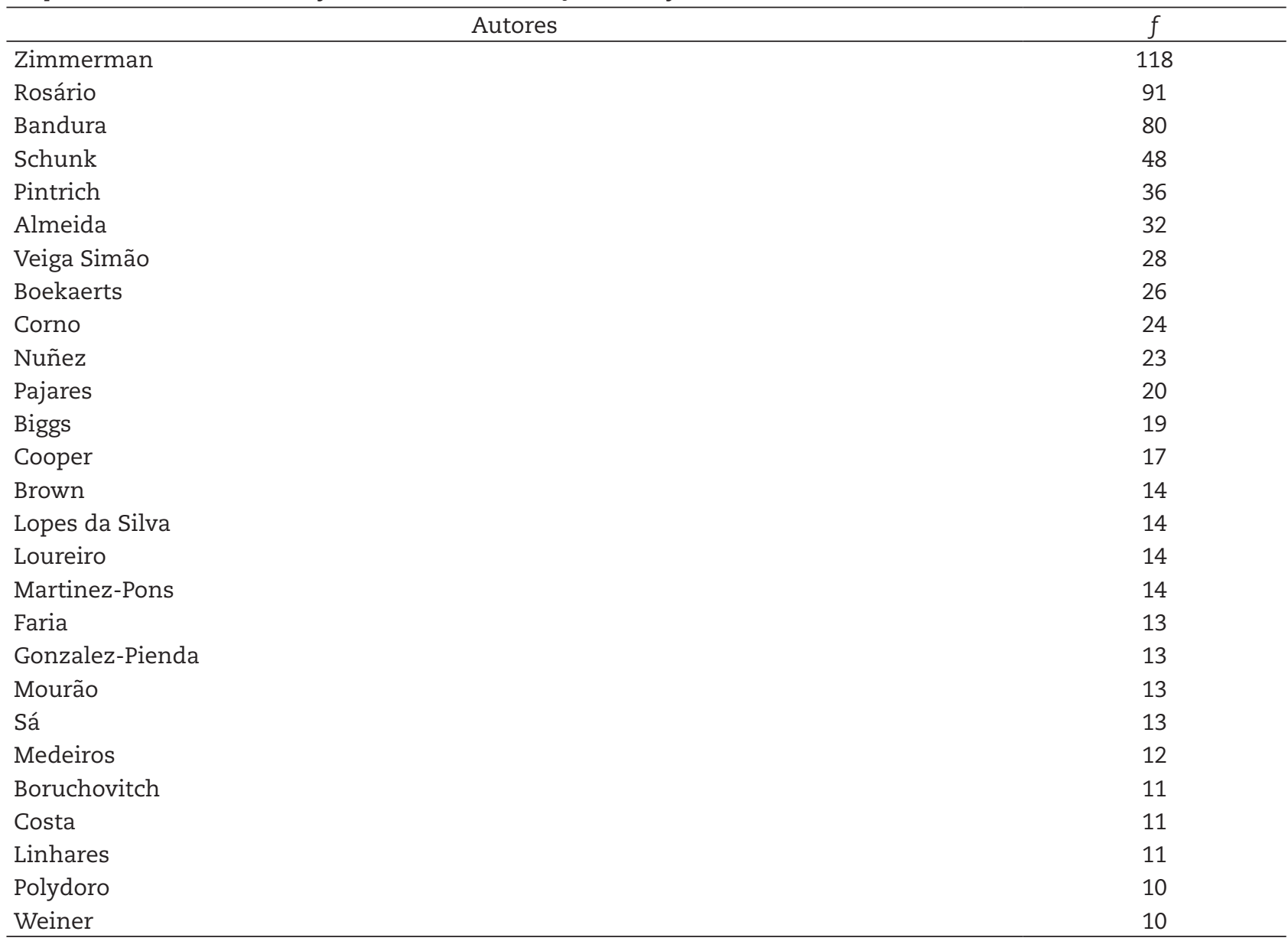

Considerando os quadrantes formados, pode-se inferir que o autor P. S. L. F. Rosário está mais vinculado ao conceito de autorregulação da aprendizagem, enquanto A. Bandura está mais ligado ao conceito de autoeficácia. Já B. J. Zimmerman é um autor que circula entre os dois temas, sendo referenciado nos trabalhos de ambos os conceitos, confirmando-o como um autor de referência e destaque no tema.

A Figura 2 apresenta uma ilustração da rede de relacionamentos entre esses autores. De maneira geral, apresentam um alto grau de interconexões, o que pode ser averiguado pela medida de densidade de rede. Essa medida é a proporção entre os relacionamentos possíveis e os relacionamentos efetivados (Neiva \& Torres, 2011). Considerando os 27 autores, a densidade da rede é de $71,5 \%$, o que pode ser qualificado como densa, em que os autores conservam muitas ligações entre si. Isso é uma característica desejável, já que apenas dois conceitos são tratados, sendo que eles estão, em sua maior parte, inseridos na mesma teoria, uma vez que a maior parte dos artigos apresenta a mesma teoria de base.

Focando na estrutura de relacionamentos, percebe-se que A. Bandura não ocupa um lugar central na rede, apesar de muito conectado e citado. Isso ocorre já que ele, como visto no escalonamento multidimensional, está fortemente ligado a um dos conceitos o que, por si só, coloca-o mais em contato com os autores que têm muitas publicações na área. L. Almeida, B. J. Zimmeman, D. H. Schunk, P. S. L. F. Rosário e S. Soares parecem ser os principais elos entre os dois conceitos, relacionando-se intensamente com os dois lados da rede. De modo geral, é possível identificar a epistemologia que fundamenta o estudo de ambos os temas tanto no Brasil quanto em Portugal. 


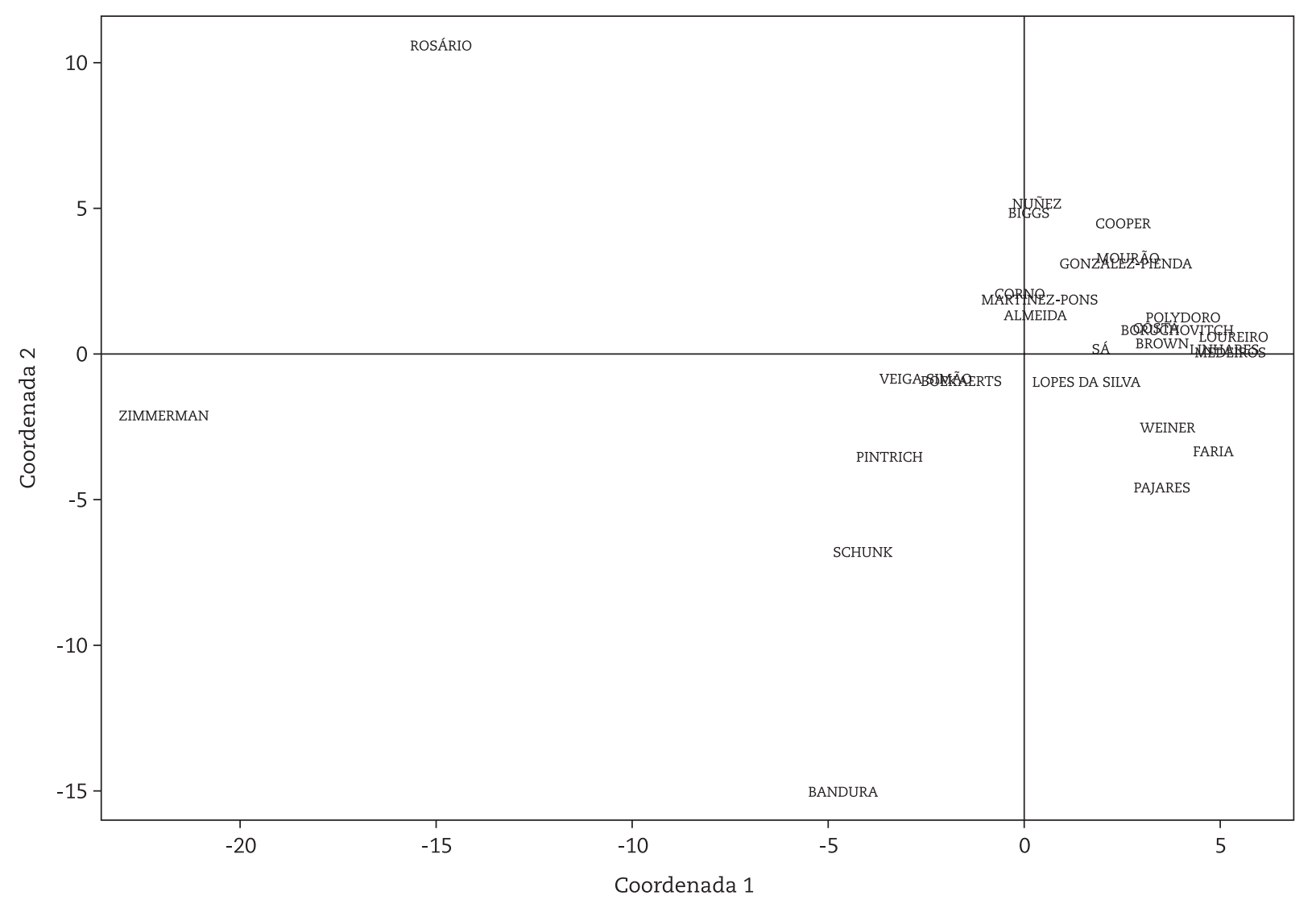

Figura 1. Representação gráfica da distância euclidiana entre os autores mais citados

A análise da produção científica de uma determinada área de estudos é um elemento útil de ordenação e estruturação do conhecimento produzido por permitir conhecer os principais autores e as referências bibliográficas centrais das disciplinas pesquisadas, como apontado por Chen et al. (2002), Población, Witter, e Silva (2006) e Witter (1999). O uso da cientometria aliada ao uso de métodos pictográficos para a representação dos relacionamentos entre as categorias (Noyons, 2001) e a análise das redes de relacionamento de acordo com o modelo de Leydesdorff $(2008 ; 2011)$ e Salvador e López-Martínez (2000) permitiu caracterizar a produção em autoeficácia e autorregulação e identificar as necessidades de investigação, bem como possíveis avanços na área.

No presente estudo, a exploração da estrutura subjacente à produção em língua portuguesa sobre a autoeficácia acadêmica e a autorregulação da aprendizagem, apontou a prevalência da Teoria Social Cognitiva, postulada por Bandura (2008) ao longo do século passado. No entanto, ele não foi o autor mais citado, posto ocupado por B. J. Zimmerman, especialmente por contribuir com ambos os construtos. Esse resultado pode revelar quanto é importante a caracterização dos elementos que compõem o processo de aprendizagem de modo a possibilitar tanto a identificação das dificuldades apresentadas pelos universitários quanto saber como intervir para buscar o sucesso acadêmico sob a perspectiva das habilidades cognitivas e sociais.

Com isso, pode-se concluir que a análise bibliográfica cumpriu seu objetivo de apontar e descrever a organização subjacente aos construtos, provendo informação útil tanto para orientar aqueles que desejam iniciar seus estudos, quanto para desenhar um quadro mais nítido do estado desse campo entre aqueles que já atuam na área.

Em termos de conclusão, a análise das publicações apontou que, em sua maioria, os dois construtos são abordados de forma independente, como já foi constatado por Maciel (2010). Dentre as limitações deste estudo está a necessidade de mais estudos de intervenção que enfoquem os dois construtos para verificar o quanto o desempenho acadêmico pode ser modificado pela inter-relação entre eles. Além disso, a baixa frequência de referências nacionais, conjugada com o pequeno número de artigos teóricos, sugere um espaço para o debate acerca dos resultados das pesquisas efetuadas em língua portuguesa. 


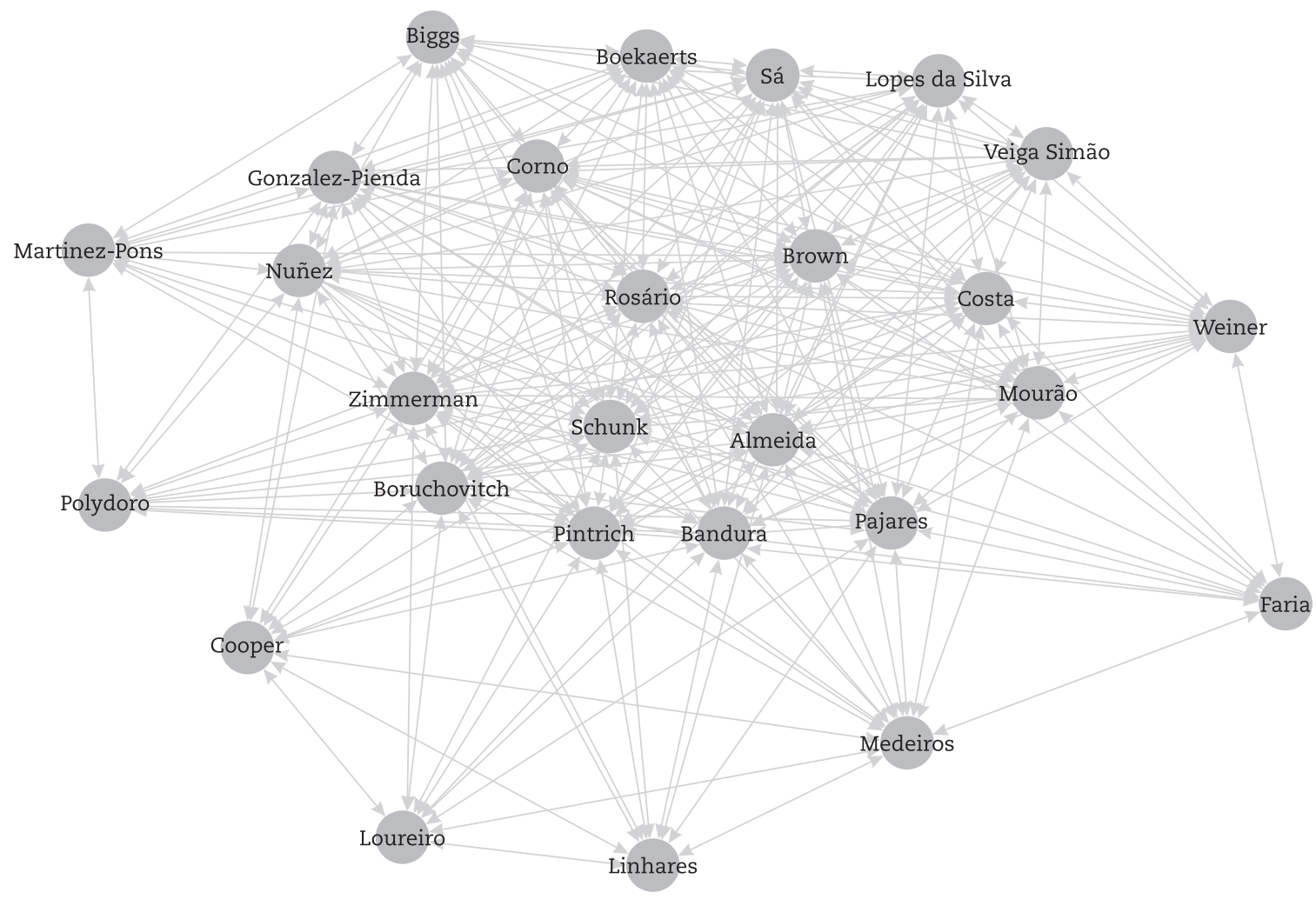

Figura 2. Representação da rede de relacionamentos entre os autores mais referenciados

\section{Referências}

Bandura, A. (1991). Self-regulation of motivation through anticipatory and self- reactive mechanisms. Em R. A. Dienstbier (Ed.) Perspectives on motivation: Nebraska symposium on motivation (pp. 69-164). Lincoln: University of Nebraska Press.

Bandura, A. (1993). Perceived self-efficacy in cognitive development and functioning. Educational Psychologist, 28, 117-148.

Bandura, A. (1997). Self-efficacy: The exercise of control. New York: W. H. Freeman.

Blickle, G. (1996). Personality traits, learning strategies, and performance. European Journal of Personality, 10, 337-352.

Boekaerts, M. (1996). Personality and the psychology of learning. European Journal of Personality, 10, 377-404.

Butts, C. T., Handcock, M. S., \& Hunter, D. R. (2012). Network: Classes for relational data. R package version 1.7-1. Irvine, CA. Recuperado de http://statnet.org/

Bzuneck, J. A. (2005). A motivação dos alunos em cursos superiores. Em M. C. R. A. Joly, A. A. A. dos Santos \& F. F. Sisto (Eds.). Questões do cotidiano universitário (pp. 217-237). São Paulo: Casa do Psicólogo.

Caprara, G. V., Fida, R., Vecchione, M., Del Bove, G., Vecchio, G. M., Barbaranelli, C., \& Bandura, A. (2008). Longitudinal analysis of the role of perceived self-efficacy for self-regulated learning in academic continuance and achievement. Journal of Educational Psychology, 100, 525-534. doi:10.1037/0022-0663.100.3.525

Casanova, D. C. G., Azzi, R. G., \& Dantas, M. A. (2010) Autoeficácia acadêmica: possibilidade para refletir sobre o ensino médio. Eccos Revista Científica, 12(1) 51-68.

Castro, M. A. S. N. (2007). Processos de autorregulação da aprendizagem: impacto de variáveis académicas e sociais (Dissertação de mestrado, Universidade do Minho, Minho, Portugal). Recuperado de http://repositorium.sdum.uminho.pt/handle/1822/7240

Chen, C., McCain, K., White, H., \& Lin, X. (2002). Mapping scientometrics (1981-2001). Proceedings of the American Society for Information Science and Technology, 39(1), 25-34.

Choi, N. (2005). Self-efficacy and self-concept as predictors of college student's academic performance. Psychology in the Schools, 42, $197-205$. doi:10.1002/pits.20048

Eilam, B., Zeidner, M., \& Aharon, I. (2009). Student conscientiousness, self-regulated learning, and science achievement: And explorative field study. Psychology in the Schools, 46(5), 420-432.

Flavell, J. H. (1979). Metacognition and cognitive monitoring: A new area of cognitive-developmental inquiry. American Psychologist, 34(10), 906-911.

Guimarães, S. E. R., \& Boruchovitch, E. (2004). O estilo motivacional do professor e a motivação intrínseca dos estudantes: uma perspectiva da teoria da autodeterminação. Psicologia: Reflexão e Crítica, 17(2), 143-150.

Joly, M. C. R. A., Cecílio-Fernandes, D., Dias, A. S., Oliveira, S. M. S. S., Bustamante, M. I., Ávila-Batista, A. C, \& Monteiro, S. C. (2014). Comparacão das versões completa e reduzida da escala de competência de estudo utilizando Rasch com universitários brasileiros. Revista de Estudios e Investigación en Psicología y Educación, 1(1), 35-43. 
Joly, M. C. R. A., \& Prates, E. A. R. (2011). Teaching and learning in information society: The importance of self-regulation and self-efficacy. Em A. Mendez-Villas (Ed.) Education in technological world: Communicating current and emerging research and technological efforts (pp. 448-456). Badajoz: Formatex.

Knijnik, L. F., Giacomoni, C. H., Zanon, C., \& Stein, L. M. (2014). Avaliação dos subtestes de leitura e escrita do teste de desempenho escolar através da teoria de resposta ao item. Psicologia: Reflexão e Crítica, 27(3), 481-490.

Leydesdorff, L. (2007). Visualization of the citation impact environments of scientific journals: An online mapping exercise. Journal of the American Society for Information Science and Technology, 58(1), 25-38.

Leydesdorff, L. (2008). On the normalization and visualization of author co-citation data: Salton's cosine versus the Jaccard index. Journal of the American Society for Information Science and Technology, 59(1), 77-85.

Leydesdorff, L. (2011). Bibliometrics/Citation networks. Em G.A. Barnett (Ed.), The encyclopedia of social networks (vol. I pp. 72-74). London: Sage.

Lopes da Silva, A., \& Sá, I. (1993). Saber estudar e estudar para saber. Porto: Porto Editora.

Lopez Lopez, W., Silva, L. M., Garcia-Cepero, M. C., Aguilar-Bustamante, M. C., \& Lopez, E. A. (2010). Panorama general de la producción acadêmica en la psicologia Colombiana indexada en PsicoRedalyc, 2005-2007. Acta Colombiana de Psicologia, 13(2), 35-46.

Maciel, A. C. M. (2010). A autoeficácia e a autorregulação em estudos sobre a aprendizagem de língua estrangeira por estudantes de ensino superior (Dissertação de mestrado, Universidade Estadual de Campinas, Campinas, Brasil). Recuperado de http://www.bibliotecadigital. unicamp.br/document/?code $=000767805$

Manly, B. (2008). Métodos estatísticos multivariados: uma introdução ( $3^{\mathrm{a}}$ ed.). Porto Alegre: Artmed.

Mayer, R. E. (2011). Towards a science of motivated learning in technology-supported environments. Educational Technology Research and Development, 59, 301-308.

Neiva, E. R., \& Torres, C. V. (2011). Psicologia social no Brasil: uma introdução. Em C. V. Torres \& E. R. Neiva (Eds.). Psicologia social: principais temas e vertentes (pp. 31-57). Porto Alegre: Artmed.

Noyons, E. (2001). Bibliometric mapping of science in a policy context. Scientometrics, 50(1), 83-98.

Pajares, F., \& Olaz, F. (2008). Teoria social cognitiva e autoeficácia: uma visão geral. Em A. Bandura, R. G. Azzi \& S. A. J. Polydoro (Eds.) Teoria social cognitiva: conceitos básicos (pp. 97-114). Porto Alegre: Artmed.

Paris, S. G., \& Winograd, P. (1990). How metacognition can promote academic learning and instruction. Em B. F. Jones \& L. Idol (Eds.) Dimensions of thinking and cognitive instruction (pp. 15-51). Hillsdale: Erlbaum.

Poblácion, D. A., Witter, G. P., \& Silva, J. F. M. (2006). Comunicação \& produção científica: contexto, indicadores e avaliação. São Paulo, SP: Angellara Editora.

Polydoro, S. A. J., \& Azzi, R. G. (2009). Autorregulação da aprendizagem na perspectiva da teoria sociocognitiva: introduzindo modelos de investigação e intervenção. Psicologia da Educação, 29, 75-94.

R Development Core Team (2012). R: A language and environment for statistical computing. R Foundation for Statistical Computing, Vienna, Austria. ISBN 3-900051-07-0. Recuperado de http://www.R-project.org/.

Revelle, W. (2012) Psych: Procedures for personality and psychological research. Northwestern University, Evanston. Recuperado de http:// personality-project.org/r/psych.manual.pdf, 1.2.1

Rosário, P. S. L. F. (2001) diferenças processuais na aprendizagem: avaliação alternativa das estratégias de autorregulação da aprendizagem. Psicologia, Educação e Cultura, 1, 87-102.

Salvador, M. R., \& López-Martínez, R. (2000). Cognitive structure of research: Scientometric mapping in sintered materials. Research Evaluation, 9(3), 189-200.

Schunk, D. H. (1995). Self-efficacy and education and instruction. Em J. E. Maddux (Ed.). Self-efficacy, adaptation and adjustment- theory, research and application (pp. 281-303). New York: Plenum Press.

Schunk, D. H., \& Ertmer, P. A. (2000) Self-regulation and academic learning: Self-efficacy enhancing interventions. Em M. Boekaerts, P. R. Pintrich \& M. Zeidner (Eds.). Handbook of Self-regulation (pp. 631-650). New York: Academic Press.

Usher, E. L., \& Pajares, F. (2007). Self-efficacy for self-regulated learning: A validation study. Educational and Psychological Measurement, 68, 443-463.

Valle, A., Cabanach, R. G., González-Pienda, J. A., Núñez, J. C., Rodríguez, S., \& Rosário, P. (2009). Perfiles motivacionales en estudiantes de secundaria: Análisis diferencial en estrategias cognitivas, estrategias de autorregulación y rendimiento académico. Revista Mexicana de Psicología, 26(1), 113-124.

Wild, F. (2011). Isa: Latent semantic analysis. R package version 0.63-3. Recuperado de http://CRAN.R-project.org/package =1sa

Witter, G. P. (1999). Metaciência e leitura. Em G. P. Witter (Eds.). Leitura: textos e pesquisas. (pp. 13 -22). Campinas, SP: Editora Alínea.

Zimmerman, B. J. (1989). A social cognitive view of self-regulated academic learning. Journal of Educational Psychology, 81(3), 329-339.

Zimmerman, B. J. (2000). Attaining self-regulation - A social cognitive perspective. Em M. Boekaerts, P. R. Pintrich \& M. Zeidner (Eds.). Handbook of self-regulation (pp. 631-650). New York: Academic Press.

Zimmerman, B. J. (2001). Models of self-regulated learning and academic achievement. Em B. J. Zimmerman \& D. H. Schunk (Eds). Self-regulated leaning and academic achievement: Theory, research, and practice (2a Ed, pp. 1-38). New York, NY: Routledge.

Zimmerman, B. J. (2013). From cognitive modeling to self-regulation: A social cognitive career path. Educational Psychologist, 48(3), 135-147.

\section{Sobre os autores}

Maria Cristina Rodrigues Azevedo Joly é doutora em psicologia do desenvolvimento humano e aprendizagem pela Universidade de São Paulo com estágio de pós-doutoramento em avaliação psicológica e educacional no Instituto de Educação da Universidade do Minho. Pesquisadora associada do Instituto de Psicologia e Programa de Pós-Graduação stricto sensu em Processos de Desenvolvimento Humano e Saúde na Universidade de Brasília.

Alexandre de Oliveira Serpa é doutorando em psicologia da Universidade São Francisco. Gerente de Pesquisa e Desenvolvimento da Hogrefe Cetepp.

Lisandra Borges é doutora em psicologia pela Universidade São Francisco. Professora no curso de Psicologia da Universidade São Francisco.

Rosana Maria Mohallem Martins é doutoranda em Psicologia na Universidade São Francisco. Professora no curso de Psicologia do Centro Universitário de Itajubá. 